\title{
Effect of different sampling designs and methods on the estimation of secondary production: A simulation
}

\author{
Mathieu Cusson, ${ }^{1}$ Jean-François Plante, ${ }^{2}$ and Christian Genest ${ }^{3}$ \\ ${ }^{1}$ Québec-Océan, Département de biologie, Université Laval, Québec, Canada \\ ${ }^{2}$ Department of Statistics, University of British Columbia, Vancouver, British Columbia, Canada \\ ${ }^{3}$ Département de mathématiques et de statistique, Université Laval, Québec, Canada
}

\begin{abstract}
This article reports the results of a simulation study designed to investigate the effect of several sampling design factors on the accuracy and precision of various estimates of secondary production. Whereas most previous studies of this sort were concerned with freshwater fauna (e.g., insects), the hypothetical population used here reflects the characteristics of marine mussels from cold-temperate and subarctic regions. It features the simultaneous presence of different cohorts, gradual recruit arrival, seasonal growth oscillation, and quadratdependent population density, as well as random individual variation both in survival and in weight gain. For this population, the percentage relative bias (PRB) and relative root mean squared error (RRMSE) of 4 classic cohort-based methods, 3 size-based methods, and several variants thereof were computed as a function of sampling frequency, distribution of sampling dates, number of quadrats sampled per occasion, inclusion or omission of the last sampling date, and coarseness of the size classes and sieve aperture. Although most methods performed reasonably well, non-negligible differences were observed among them. A version of Allen's curve technique and a mass-specific growth rate method gave the best results for cohort- and size-based method groups, respectively. Sampling effort, in terms of both frequency of sampling and number of samples per date, had the largest documented influence on both PRB and RRMSE. Recommendations are made for the best compromises between methods and sampling designs to achieve reliable production estimates for populations with similar characteristics.
\end{abstract}

\section{Introduction}

In marine studies, the secondary production of macrobenthic populations can be estimated in a number of ways. Classic techniques can be broadly classified as cohort- and size-based. Prominent in the first group are the Allen-curve, incrementand removal-summation, and instantaneous-growth methods. The second group consists mainly of the size-frequency, massspecific growth rate, and mass-specific mortality rate methods.

These estimation techniques, and numerous variants thereof, have been described, e.g., by Benke (1984), Crisp (1984), and Rigler and Downing (1984). They have been extensively compared through simulations, most notably by Cushman et al. (1978), Lapchin and Neveu (1980), and Morin et al. (1987). These and other authors examined how various estimates were affected by different assumptions on the

\footnotetext{
Acknowledgments

Partial funding in support of this work was provided by the Natural Sciences and Engineering Research Council of Canada, and by the Fonds québécois de la recherche sur la nature et les technologies.
}

growth and mortality curves, as well as by sampling effort. Their hypothetical populations, which were alternately assumed to be synchronous or not, were modeled on freshwater species having a lifespan of 1 year or less.

This article revisits the issue and expands on the comparison of the above production estimates, and several of their variants introduced in the literature, using a hypothetical population of mussels characterized by a number of realistic factors. Most prominent among them are the simultaneous presence of different cohorts, quadrat-dependent population density, seasonal growth oscillation, gradual recruit arrival, and random individual variation both in survival and in weight gain.

The simulations presented herein are also novel in that several aspects of the sampling design are considered, namely the number of sampling occasions (either regularly spaced or concentrated in the growing season), quadrats sampled per occasion, and use of two different sieves. Following Cushman et al. (1978), the bias and variance of the various estimators are compared to the exact production obtained by summing all individual body-mass gains, rather than to a theoretical Allen curve which may or may not reflect reality. 


\section{Materials and methods}

When faced with the problem of estimating secondary production, a biologist must make a choice among various sampling conditions and different estimation methods. The purpose of this article is to investigate the relative impact of these two major factors in the context of a common intertidal macrobenthic invertebrate population such as Mytilus spp.

To guide the users in their choices, the most informative way to proceed is to construct a hypothetical but realistic population, for which the exact production can be determined. Such a virtual universe is described under "Hypothetical population," in which the variable weight gains observed over the course of each individual's lifetime can be tallied.

Given this fixed, finite population and its exact secondary production, the variation in the production estimates then depends exclusively on the choices of sampling design and estimation technique. Bias and precision in such estimates can be determined accurately by (virtually) drawing repeatedly from the fixed population.

Seven standard, cohort- and size-based, estimators of secondary production are briefly reviewed under "Estimation techniques," and 13 of their variants that are also included in this article are presented in the Appendix. Six of the most common sampling factors considered in production studies are listed under "Simulation scheme."

Hypothetical population-A fictitious population of more than 236 million individuals was constructed whose characteristics mimic as closely as possible the life cycle of mussels (Mytilus spp.) in the St. Lawrence Estuary. The intertidal population that served as a model is located at Pointe-Mitis $\left(48^{\circ} 41^{\prime} \mathrm{N}, 68^{\circ} 02^{\prime} \mathrm{W}\right)$, near Rimouski (Québec). Its length-mass relationship was described by Fréchette and Bourget (1985), and its spatial distribution is based on raw data analyzed by Cusson and Bourget (2005).

The universe was divided into 3,280,000 quadrats of $10 \mathrm{~cm}$ $\times 10 \mathrm{~cm}$. Based on the data of Cusson and Bourget (2005), approximately $8.2 \%$ of these quadrats should be empty. Whether a quadrat is empty or not was determined randomly. Given that the sampled quadrats are to be chosen at random, the presence of spatial heterogeneity or aggregation patterns in the population need not be further modeled.

In each of the non-empty quadrats, it was assumed that the total number of mussels, all ages confounded, varied according to a log-normal distribution with mean $\mu=120.7$ and standard deviation $\sigma=167.9$, in accordance with the data of Cusson and Bourget (2005). Each mussel in a given quadrat was then assigned randomly to a cohort using a multinomial distribution whose expected frequencies per age class were derived from an exponential distribution with mean $10 / 7 \approx 1.43$ years; this corresponds to a mortality $Z$ of 0.7 . Within each age class, the survival time of each mussel was randomly determined according to the same exponential model. It was thus possible to tell, on any given day, whether a particular mussel was alive or dead.
For added realism, recruits were assumed to settle gradually. If $s$ denotes the day of the year on which a given mussel settles (with $s=1$ on July 1), it was assumed that $s$ follows a lognormal distribution with mean $v=65.2$ days and standard deviation $\tau=30.6$. This way, the peak settlement season occurs in early September, with $85-90 \%$ of recruits arriving between August 1 and October 31.

For a mussel settled at time $s$, the relation at age $t$ (in days) between its flesh dry weight $W(t, s)$ in grams and its average length $L(t, s)$ in $\mathrm{cm}$ was taken to be

$$
W(t, s)=0.0145 \times L(t, s)^{2.42},
$$

as per Fréchette and Bourget (1985). The growth function $L(t, s)$ was taken to follow a Gompertz curve. A seasonal effect was also built in to growth using an oscillatory function defined by

$$
\omega(t, s)=\frac{365}{2 \pi}\left[\sin \left\{\frac{2 \pi}{365}(t+s-31)\right\}-\sin \left\{\frac{2 \pi}{365}(s-31)\right\}\right] .
$$

More specifically, the average length at time $t$ of a mussel settled at time $s$ was given by

$$
L(t, s)=10^{a[1-\exp \{b(t / 365-c)+d \omega(t, s)\}]}-10^{a\{1-\exp (-b c)\}},
$$

where

$$
a=0.838, b=-0.584, c=1.77, d=-0.0016 .
$$

The choice of the Gompertz curve, and its associated parameters, is justified by the work of Bayne and Worrall (1980).

The general shape of this complex but realistic growth curve is depicted in Fig. 1, in terms of both average length (upper panel) and average flesh dry weight (lower panel) as a function of time, for a mussel born July 1 .

As a final ingredient, mussel-to-mussel variation in length was introduced through a cumulative daily random component which was added to $L(t, s)$ using a normal distribution with zero mean and standard deviation $6.5 \times 10^{-3} \mathrm{~cm}$. This choice of parameters guaranteed that among mussels 8 years of age, $95 \%$ of individuals had a length of $6.52 \pm 0.69 \mathrm{~cm}$.

Altogether, the assumptions made on this hypothetical population of mussels led to a mean mussel density of 7201 individuals $\mathrm{m}^{-2}$ and an average flesh dry weight production of $351.1 \mathrm{~g} \mathrm{~m}^{-2} \mathrm{yr}^{-1}$. Using the conversion factor of $21.15 \mathrm{~kJ} /$ gSFDW of Brey (2004), this corresponds to $7425.6 \mathrm{~kJ} \mathrm{~m}^{-2} \mathrm{yr}^{-1}$, which is representative of the production of a natural population of mussels.

Estimation techniques - Seven approaches to the estimation of the secondary production were considered, which can be broadly classified into two groups:

- Cohort-based methods: increment summation (IS), removal summation (RS), instantaneous-growth (IG), and Allen's curve (AC);

- Size-based methods: size frequency (SF), mass-specific growth rate (GR), and mass-specific mortality rate (MR). 

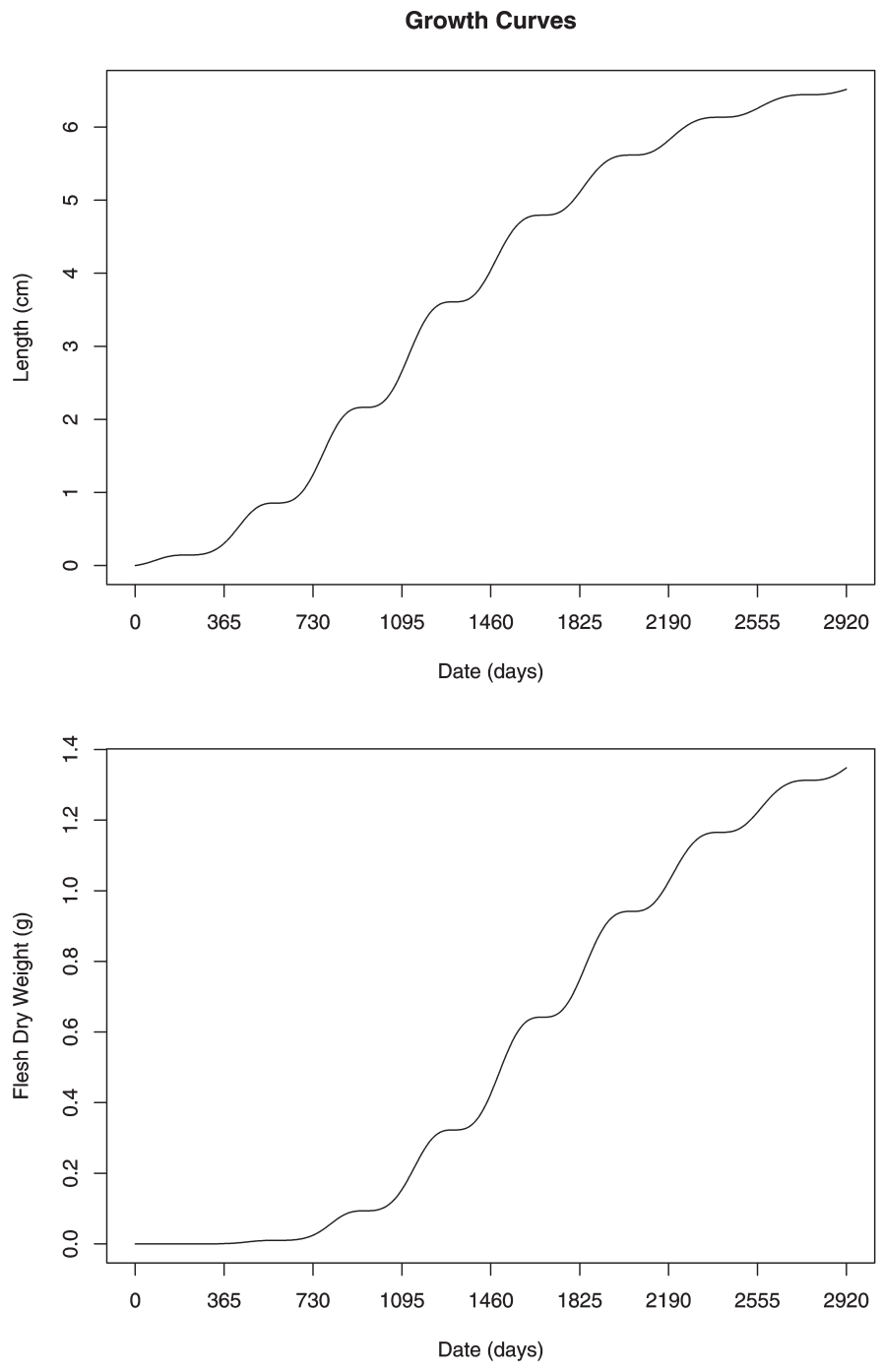

Fig. 1. Length (upper panel, in $\mathrm{cm}$ ) and flesh dry weight (lower panel, in g) of a typical mussel from the hypothetical population, as modeled over 8 years.

These 7 estimation techniques are briefly recalled below; 13 variants that were included in the simulation study are also described in Web Appendix 1. The following notation is used in the sequel: $I$ is the number of age classes ( 8 in the study), $K$ is the number of size classes, $\left(L_{k}, L_{k+1}\right)$ represents the $k$ th size class, $T$ is the number of sampling occasions, and $T_{1}, \ldots, T_{T}$ denote the sampling dates. Let also

$N_{\text {tik }}=$ average over all sampled quadrats of the number of individuals from cohort $i$ whose length falls between $L_{k}$ and $L_{k+1}$ at time $T_{t^{\prime}}$

$B_{t i k}=$ flesh dry weight in grams averaged per sampled quadrat for individuals from cohort $i$ whose length falls between $L_{k}$ and $L_{k+1}$ at time $T_{t}$

$$
N_{t i \bullet}=\sum_{k=1}^{K} N_{t i k}, \quad B_{t i \bullet}=\sum_{k=1}^{K} B_{t i k}, \quad W_{t i \bullet}=B_{t i \bullet} / N_{t i \bullet},
$$

$$
N_{\bullet \bullet k}=\frac{1}{I T} \sum_{t=1}^{T} \sum_{i=1}^{I} N_{t i k}, \quad B_{\bullet \bullet k}=\frac{1}{I T} \sum_{t=1}^{T} \sum_{i=1}^{I} B_{t i k}, \quad W_{\bullet \bullet k}=B_{\bullet \bullet k} / N_{\bullet \bullet k} .
$$

IS-1 Classic increment summation method: Total production is estimated by $P=P_{1}+\ldots+P_{I}$, where

$$
P_{i}=\sum_{t=1}^{T-1}\left(N_{(t+1) i \bullet}+N_{t i \bullet}\right)\left(W_{(t+1) i \bullet}-W_{t i \bullet}\right) / 2 .
$$

RS Removal summation method: This technique, which is well known to yield exactly the same answer as IS-1, estimates $P=P_{1}+\ldots+P_{I}$ with

$$
P_{i}=\left(B_{T i \bullet}-B_{1 i \bullet}\right)+\sum_{t=1}^{T-1}\left(N_{t i \bullet}-N_{(t+1) i \bullet}\right)\left(W_{(t+1) i \bullet}+W_{t i \bullet}\right) / 2 .
$$

IG-1 Classic instantaneous-growth method: $P=P_{1}+\ldots+P_{I}$, where

$$
P_{i}=\sum_{t=1}^{T-1}\left(B_{(t+1) i \bullet}+B_{t i \bullet}\right) \log \left(\frac{W_{(t+1) i \bullet}}{W_{t i \bullet}}\right) / 2 .
$$

When it occurred that no individuals were sampled on some occasions, linear regression was used to infer the number of individuals and their average weight at that time. Two curves were fitted, namely $\log \left(N_{t i \bullet}\right)=\alpha+\beta T_{t}$ and $\log \left(W_{t i \bullet}\right)=\alpha^{\star}+\beta^{\star} T_{t}$. This imputation was needed to avoid zeros in the logarithmic term of the sum.

AC-1 Classic Allen's curve method: For a fixed age class $i$, the curve

$$
\log \left(N_{t i \bullet}\right)=\alpha_{i}+\beta_{i} \log \left(W_{t i \bullet}\right)
$$

is fitted. Writing $W_{\min i}$ and $W_{\max i}$ for the smallest and largest values in the set $\left\{W_{t i \bullet}: t=1, \ldots, T\right\}$, respectively, one then computes $P=P_{1}+\ldots+P_{I}$ with

$$
P_{i}=\frac{e^{\alpha_{i}}}{\beta_{i}+1}\left(W_{\max i}^{\beta_{i}+1}-W_{\min i}^{\beta_{i}+1}\right) \text {. }
$$

SF-1 Classic size-frequency method:

$$
P=K \sum_{k=1}^{K-1}\left(N_{\bullet \bullet(k+1)}-N_{\bullet \bullet k}\right)\left(W_{\bullet \bullet(k+1)}+W_{\bullet \bullet k}\right) / 2 .
$$

GR-1 Classic mass-specific growth rate method, based only on data:

$$
P=K \sum_{k=1}^{K-1} B_{\bullet \bullet} \log \left(\frac{W_{\bullet \bullet(k+1)}}{W_{\bullet \bullet k}}\right) .
$$

MR-1 Classic mass-specific mortality rate method:

$$
P=K \sum_{k=1}^{K-1} B_{\bullet \bullet k} \log \left(\frac{N_{\bullet \bullet(k+1)}}{N_{\bullet \bullet k}}\right) .
$$

Simulation scheme-A Monte Carlo study was carried out to assess the performance of the 7 standard estimators of secondary production and 13 of their variants with respect to the following factors:

A. number $T$ of sampling occasions $(5,7$, or 13 per year, including July 1);

B. inclusion or not of June 30 as the last sampling occasion 


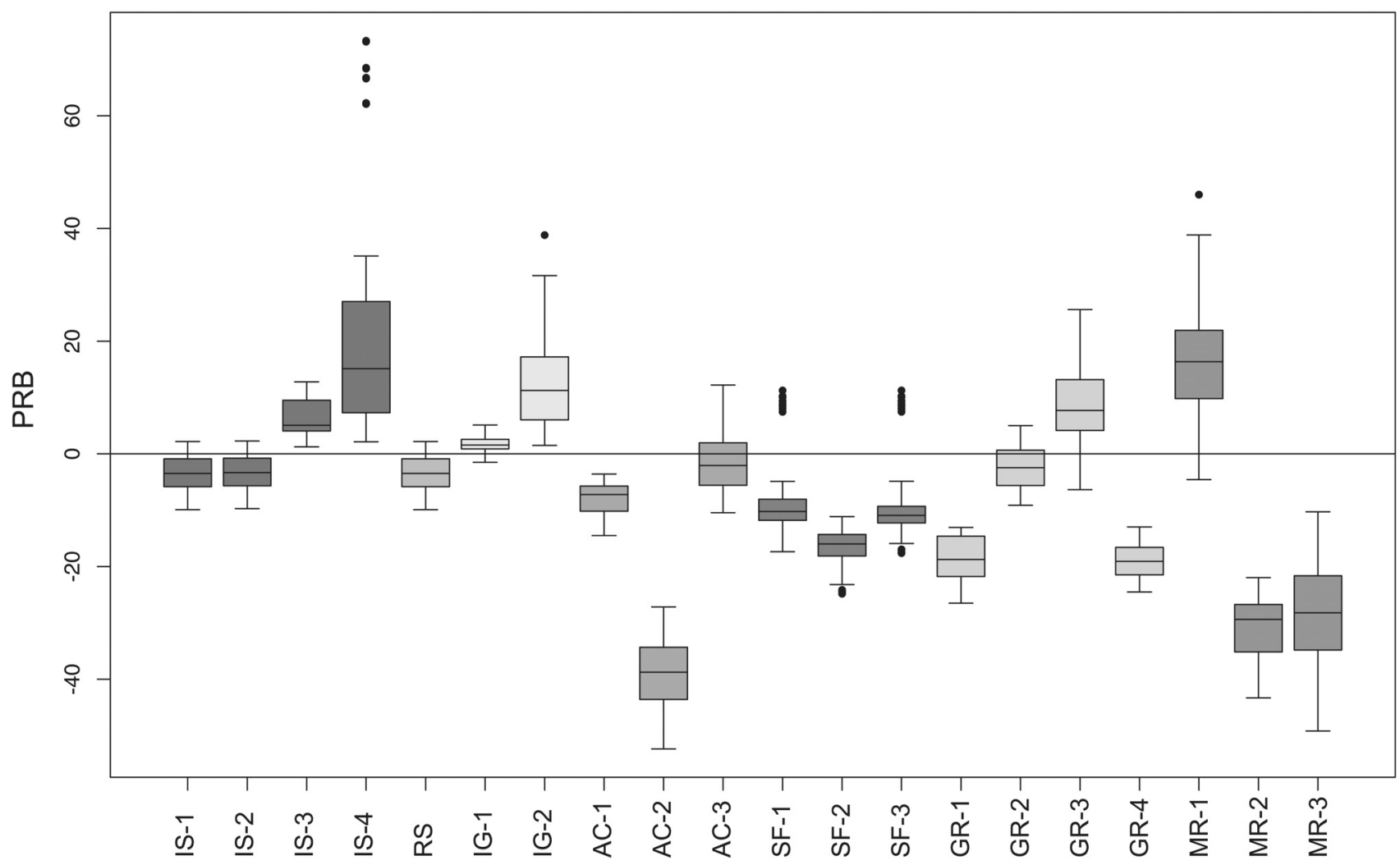

Fig. 2. Observed PRB of 20 methods of estimating secondary production in 72 (or 216) combinations of factors A-F defined under "Simulation scheme"; each point is based on 1000 repetitions. The middle line and extremities of the box are at the 25th, 50th, and 75th percentiles; the maximum length of each whisker is 1.5 times the interquartile range; outliers are shown individually.

(the data collected on the following July 1 serving as a surrogate if needed);

C. distribution of other sampling dates, either evenly throughout the year or concentrated in the growing season, so that the growth in length of an average individual from cohort no 4 (arbitrarily chosen, but median) is the same between any 2 consecutive sampling dates in the year;

D. sampling effort $(5,10$, or 15 quadrats per sampling date);

E. screen aperture of the sieve $(0.5$ or $1 \mathrm{~mm})$, all individuals with length inferior to the mesh size being ignored;

F. when applicable, number $K$ of size classes used $(10,15$, or 30): 29 equal classes were considered from 0 to $7.25 \mathrm{~cm}$, the 30th class containing all individuals exceeding $7.25 \mathrm{~cm}$ in length; adjacent classes were combined 2 or 3 at a time to yield 10 or 15 classes.

In total, there are thus $3 \times 2 \times 2 \times 3 \times 2=72$ combinations of factors A-E to be considered for cohort-based methods, and 3 times more, i.e., 216, when factor $\mathrm{F}$ is added for size-based estimators. Each of them, representing a different scenario, was repeated 1000 times, resulting in estimated values $\hat{\theta}_{1}, \ldots, \hat{\theta}_{1000}$ of the exact production $\theta=351.1 \mathrm{~g} \mathrm{~m}^{-2} \mathrm{yr}^{-1}$. The latter value was determined by monitoring, from settlement to death, the individual growth of all mussels in the population, and by taking an average of this exact production per quadrat. This then allowed for the determination of the percent relative bias

$$
\mathrm{PRB}=\frac{100}{\theta} \times \frac{1}{1000} \sum_{i=1}^{1000}\left(\hat{\theta}_{i}-\theta\right),
$$

and the relative root mean squared error

$$
\text { RRMSE }=\frac{100}{\theta} \times \sqrt{\frac{1}{1000} \sum_{i=1}^{1000}\left(\hat{\theta}_{i}-\theta\right)^{2}} .
$$

Both quantities are standard measures of the accuracy and precision of an estimator; in each case, the smaller (in absolute value), the better. Note that as all individuals in a sampled quadrat are measured, there is no within-quadrat sampling error in the results presented below.

\section{Assessment}

General comparison of methods-Figure 2 shows boxplots of the percent relative bias (PRB) of the 20 estimators of secondary production considered in the study. Each boxplot summarizes the variability in PRB observed across the 72 combinations of factors for a specific method; for size-based techniques, the number of scenarios is 216 . The following observations can be made from Fig. 2: 


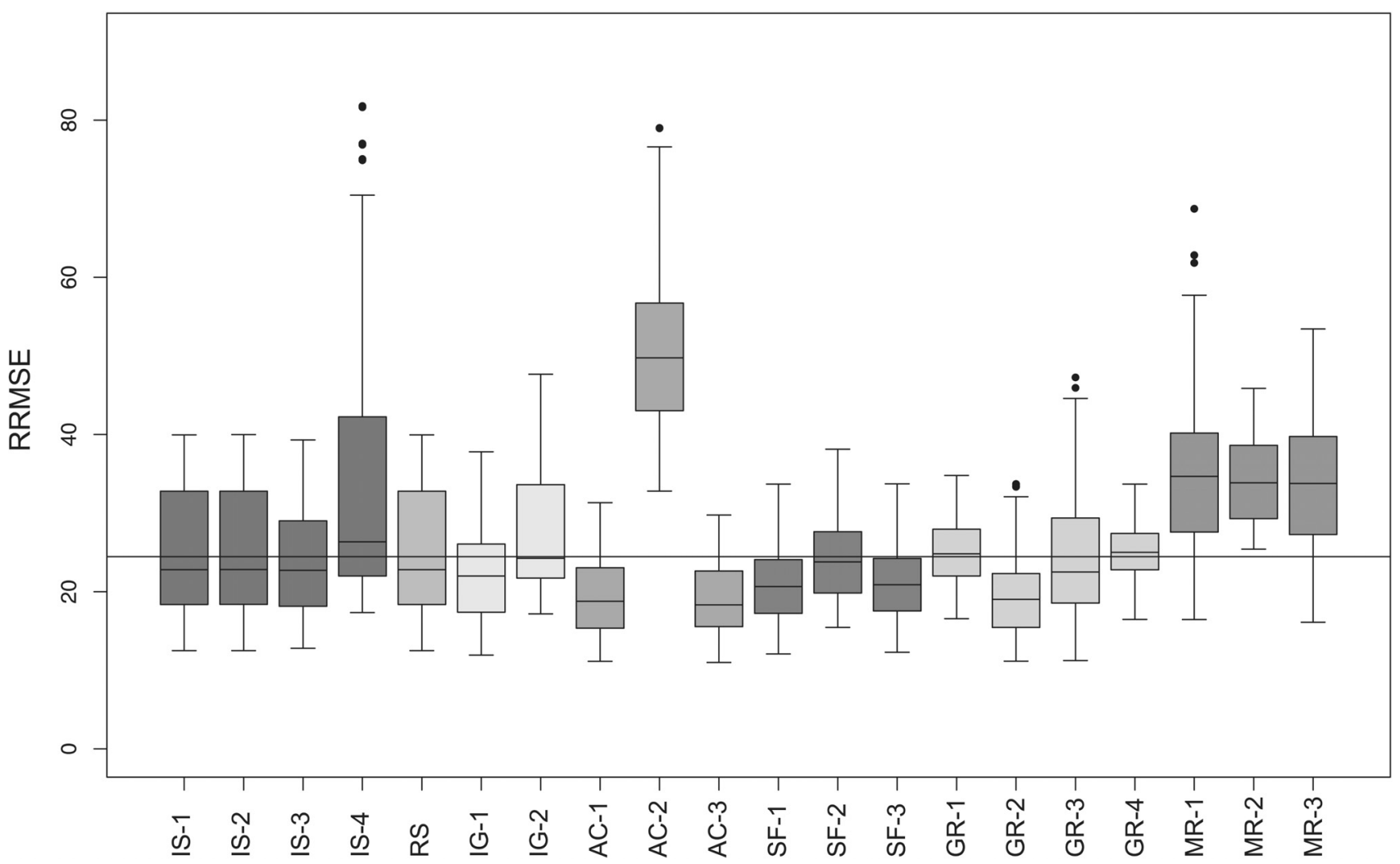

Fig. 3. Observed RRMSE of 20 methods of estimating secondary production in 72 (or 216) combinations of factors A-F defined under "Simulation scheme"; each point is based on 1000 repetitions. The central line represents the median of all observed values of the RRMSE.

1. All estimators exhibit a non-negligible bias except possibly IS-1 = RS, IS-2, IG-1, AC-3, and GR-2.

2. While IG-1 is closest to being unbiased, variant IG-2 is biased upward.

3. AC-3 is the only Allen-curve type estimator that is nearly unbiased.

4. Methods SF-1, SF-2, and SF-3 consistently underestimate production.

5. Among size-based methods, only GR-2 is approximately unbiased: whereas GR-3 and MR-1 overestimate $\theta$, all others underestimate it.

6. The PRB of IS-4, SF-1, and SF-3 is greatly affected by some combinations of factors. Note in particular that the 8 outstanding values on the boxplot for IS-4 correspond to scenarios with 13 dates and 5 quadrats per date, whereas 12 isolated points on the boxplot for SF-1 and SF-3 correspond to 10 classes, 13 dates, and a 1-mm screen aperture.

Displayed in Fig. 3 are boxplots illustrating the variation in relative root mean squared error (RRMSE) across the 72 (or 216) combinations of factors for each method. The central line represents the median of all observed values of the RRMSE.

The main observations that can be derived from Fig. 3 are as follows:
7. Methods with low RRMSE are AC-1, AC-3, SF-1, SF-3, and GR-2. 8. Methods with high RRMSE are IS-4, AC-2, MR-1, MR-2, and MR-3.

9. The RRMSE of IS-4, GR-3, and MR-1 deteriorates considerably under some combinations of factors. In particular, the outstanding values for MR-1 correspond to scenarios with 30 classes, 5 sampling occasions, and 5 quadrats per sampling date. Combining observations 1 through 9, it would seem, therefore, that AC-3 and GR-2 are the only methods that feature both small absolute PRB and RRMSE across all scenarios. Table 1 compares the PRB and RRMSE of these 2 options to the 18 other estimators considered. Surprisingly, perhaps, this table shows that the classic versions of the 7 methods are either best or close to best in their group. Therefore, additional comparisons based on individual factors are restricted to IS-1, IG-1, AC-1, AC-3, SF-1, and GR-2.

Effect of sampling effort on performance-Figure 4 shows the observed PRB of estimators IS-1, IG-1, AC-1, AC-3, SF-1, and GR-2 as a function of factors $A$ and D, i.e., the number $T$ of sampling occasions (5, 7, and 13 dates per year), and the sampling effort $(5,10$, or 15 quadrats per date). For the cohortbased methods, each boxplot depicts the variation observed in the simulation over the remaining 8 scenarios corresponding 
Table 1. Observed PRB and RRMSE per quadrat of 20 different methods of estimating secondary production.

\begin{tabular}{lrccrc}
\hline Method & \multicolumn{1}{c}{ PRB } & RRMSE & Method & PRB & RRMSE \\
\hline IS-1 = RS & -3.75 & 26.03 & SF-1 & -9.19 & 22.14 \\
IS-2 & -3.63 & 26.04 & SF-2 & -16.36 & 25.42 \\
IS-3 & 6.33 & 24.97 & SF-3 & -9.87 & 22.32 \\
IS-4 & 21.06 & 38.44 & & & \\
IG-1 & 1.67 & 23.69 & GR-1 & -18.66 & 25.58 \\
IG-2 & 12.56 & 28.39 & GR-2 & -2.47 & 20.62 \\
& & & GR-3 & 8.29 & 24.97 \\
& & & GR-4 & -19.03 & 25.53 \\
AC-1 & -7.88 & 20.06 & MR-1 & 16.55 & 36.46 \\
AC-2 & -38.90 & 51.34 & MR-2 & -30.38 & 34.61 \\
AC-3 & -1.47 & 19.50 & MR-3 & -28.02 & 34.51 \\
\hline
\end{tabular}

The figures for cohort-based methods are averaged over 72 combinations of factors A-E; the figures for size-based methods are averaged over 216 combinations of factors $\mathrm{A}-\mathrm{F}$.

to factors B, C, E; for size-based methods, factor $\mathrm{F}$ raises the number of scenarios to 24 .
For methods IS-1, AC-1, AC-3, and SF-1, the PRB approaches zero as the number of quadrats per date increases. The same is possibly true for IG-1, but is less obvious from the graph. By contrast, method GR-2 seems to be relatively insensitive to the sampling effort per occasion (factor D). Given a sampling effort in terms of quadrats per date, an increase in the number of sampling occasions (factor A) does not appear to reduce bias significantly, except for methods AC-1 and SF-1. Note that when method GR-2 is applied on data gathered on 13 occasions per year, the PRB increases somewhat (in absolute value) but is much less affected by the remaining factors (B, C, E, F).

Figure 5 shows the observed RRMSE of the same 6 estimators as a function of factors $\mathrm{A}$ and $\mathrm{D}$. Here, an increase in either the number of dates per year or the number of quadrats per sampling occasion (or both) results in an obvious reduction of the RRMSE. Overall, therefore, the combined accuracy and precision of all these methods improves with sampling effort. Moreover, since nearly all boxplots are very narrow, one can conclude that once factors A and D are taken into account, the RRMSE of the 6 methods is nearly insensitive to factors B, C, E, and F.

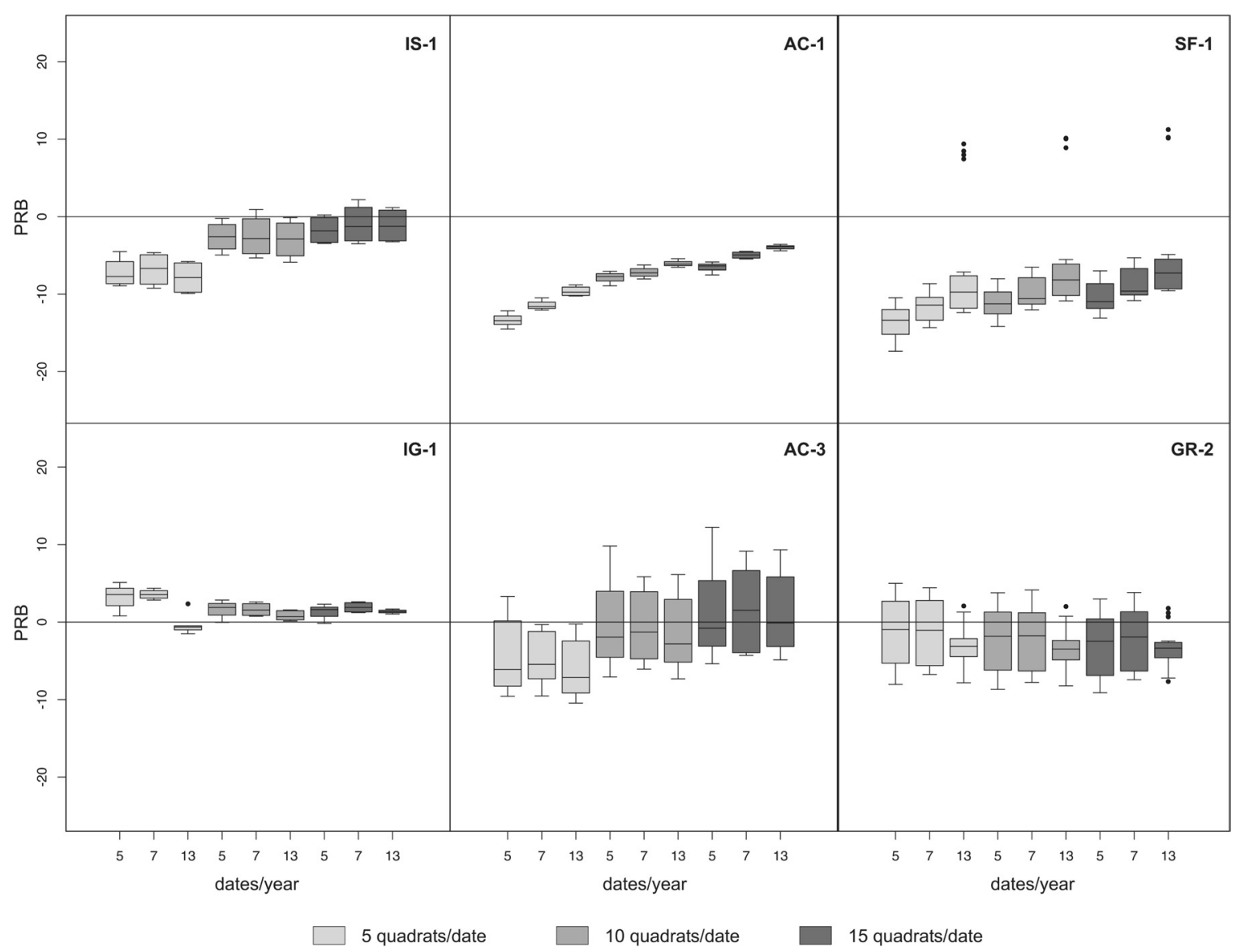

Fig. 4. Observed PRB of 6 estimators of secondary production. The 9 boxplots of each panel correspond respectively to 5, 10, and 15 quadrats for 5 , 7, and 13 sampling dates per year. Each boxplot depicts variation over the remaining 8 (or 24) combinations of factors B, C, E, and F. 


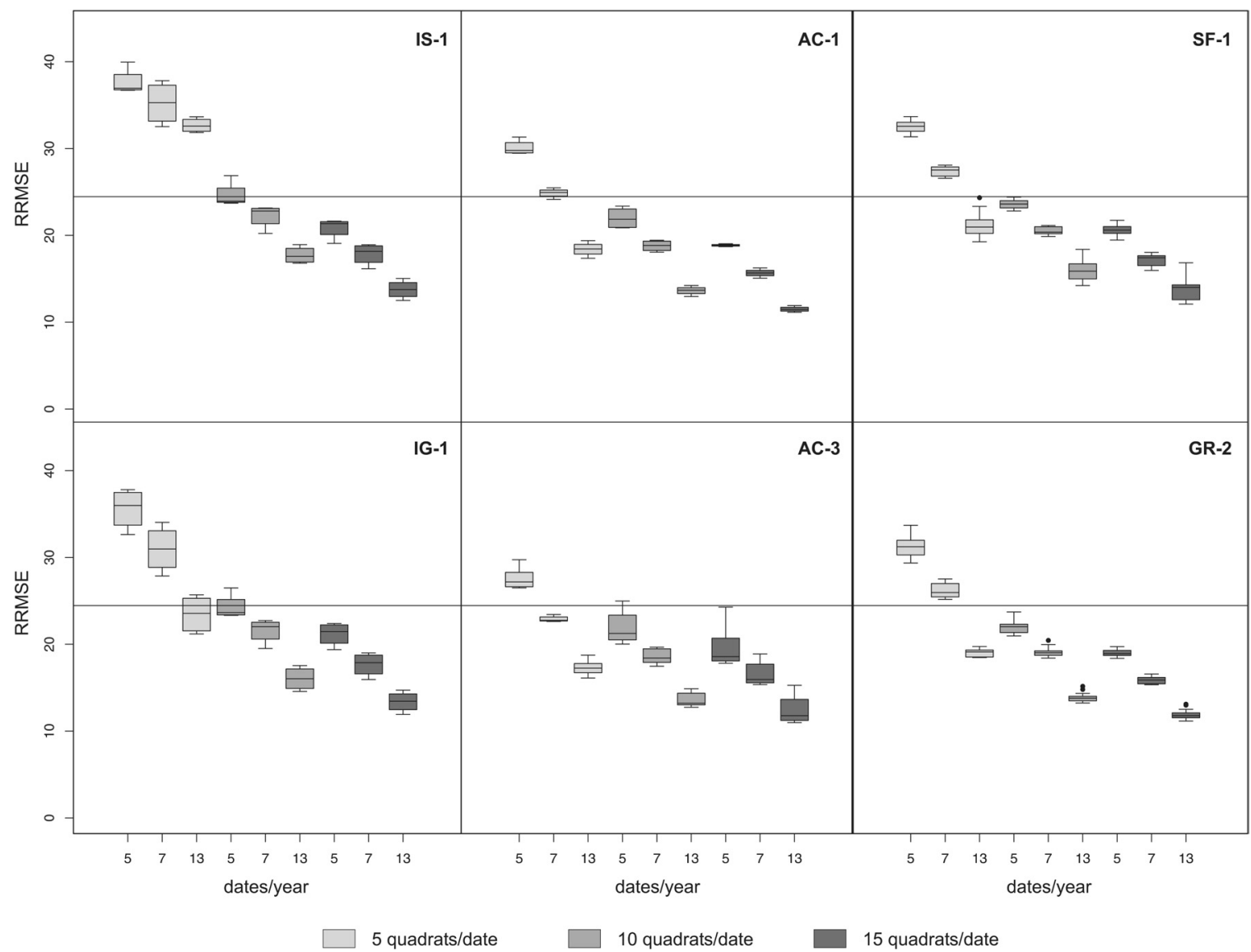

Fig. 5. Observed RRMSE of 6 estimators of secondary production. The 9 boxplots of each panel correspond respectively to 5,10 , and 15 quadrats for 5,7 , and 13 sampling dates per year. Each boxplot depicts variation over the remaining 8 (or 24) combinations of factors B, C, E, and F. The central line represents the median of all observed values of the RRMSE.

Effect of optimizing sampling dates-Depicted in Figs. 6 and 7 are the observed PRB and RRMSE of estimators IS-1, IG-1, AC-1, AC-3, SF-1, and GR-2 as a function of factors B and $\mathrm{C}$, i.e., depending on whether the sampling schedule is optimized or not and includes June 30 or not. For the cohort-based methods, each boxplot depicts the variation observed in the simulation over the remaining 18 scenarios corresponding to factors A, D, and E; for size-based methods SF-1 and GR-2, factor F raises the number of scenarios to 54 .

The PRB of the 6 estimators seems relatively unaffected by these 2 factors, except possibly IS- 1 and AC-3. The former appears to perform somewhat better when June 30 is included, while the PRB of the latter is less biased but more variable when the sampling dates are evenly distributed throughout the year. Similarly, the RRMSE of the 6 methods appears basically unaffected, whether June 30 is included or not. However, for methods IS-1, IG-1, and AC-3, there appears to be a slight advantage to the use of optimized sampling occasions in terms of overall accuracy.

Effect of sieve choice-Table 2 shows the effect of factor E (screen aperture of the sieve) on the observed PRB and RRMSE of estimators IS-1, IG-1, AC-1, AC-3, SF-1, and GR-2. The figures for the first 4 (cohort-based) methods are averaged over 36 combinations of factors A through D; the figures for the last 2 (size-based) methods are averaged over 108 combinations, due to the inclusion of factor $\mathrm{F}$.

It appears from Table 2 that the sieve's screen aperture has a negligible effect on both the PRB and RRMSE of the estimators, except for SF-1 and AC-3. In the former case, increasing the mesh size reduces bias; in the latter case, it increases bias in absolute value but appears to increase the precision of AC-3, as indicated by a reduction of the RRMSE.

Effect of number of size classes-Reported in Table 3 are the PRB and RRMSE of the 10 size-based methods of estimating secondary production, as a function of factor F, i.e., the number of size classes used to perform the calculations $(10,15$, or 30). The results represent averages over the 5 other factors, which form 72 different scenarios.

Looking at Table 3, the various methods can be classified into 3 groups, namely: 


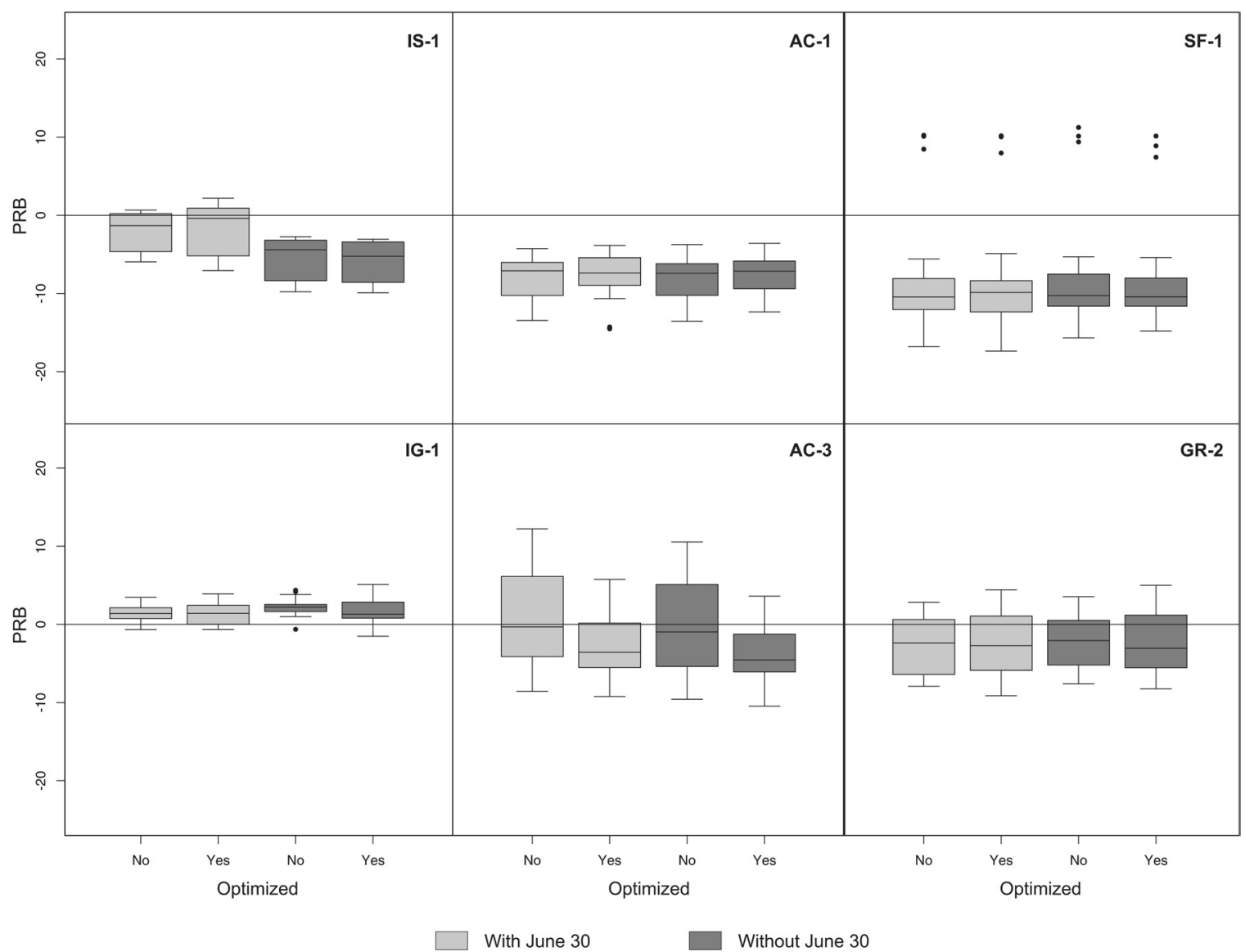

Fig. 6. Observed PRB of 6 estimators of the secondary production. The 4 boxplots of each panel correspond to optimized and non-optimized sampling schedules including June 30 or not. Each boxplot depicts variation over the remaining 18 (or 54) combinations of factors A, D, E, and F.

i. Methods for which both the PRB and RRMSE improve as the number of classes increases: GR-1, GR-4, and MR-2.

ii. Methods for which either the PRB or the RRMSE (and possibly both) deteriorates as the number of classes increases: SF-1, SF-3, GR-2, GR-3, and MR-3.

iii. Other methods: SF-2 and MR-1.

Note that in all cases, method GR-2 is actually that whose absolute PRB and RRMSE are smallest of all size-based techniques, irrespective of the number of classes; its classification into group ii is only justified by the fact that the PRB increases in absolute value with the number of size classes.

\section{Discussion}

The observations made in the present simulation study are, strictly speaking, restricted to the hypothetical population of mussels described above. However, they may be taken to apply more generally to species featuring similar characteristicslong life span, high biomass, large length or body mass, oscillatory growth, etc.

Observations and recommendations concerning methodsUnder the circumstances delineated by the hypothetical population of mussels described above, which are fairly com- mon in marine environments, the best choice among cohortbased methods appears to be Allen's curve AC-3, which exhibits both small bias and variability. If for some reason it is deemed inappropriate to merge cohorts, e.g., if the year-toyear variation in recruitment is very high, then AC-1 would constitute a reasonable alternative. Among size-based methods, GR-2 is definitely the best option (of those considered). Size-frequency methods SF-1 and SF-3 may be regarded as reasonable substitutes.

More generally, the following methodological considerations should be borne in mind when computing secondary production:

1. For mussel-like populations, the removal-summation method (RS or IS-1) is not necessarily the best choice, contra Cushman et al. (1978). Note, however, that the recommendation of these authors was based on the stability of this technique when applied to populations with widely varying characteristics.

2. The negligible difference observed in the simulation between IS-1 and Siegismund's adjustment IS-2 is likely due to the relatively low biomass of the newborn cohort. This adjustment may still be worthwhile when the first 


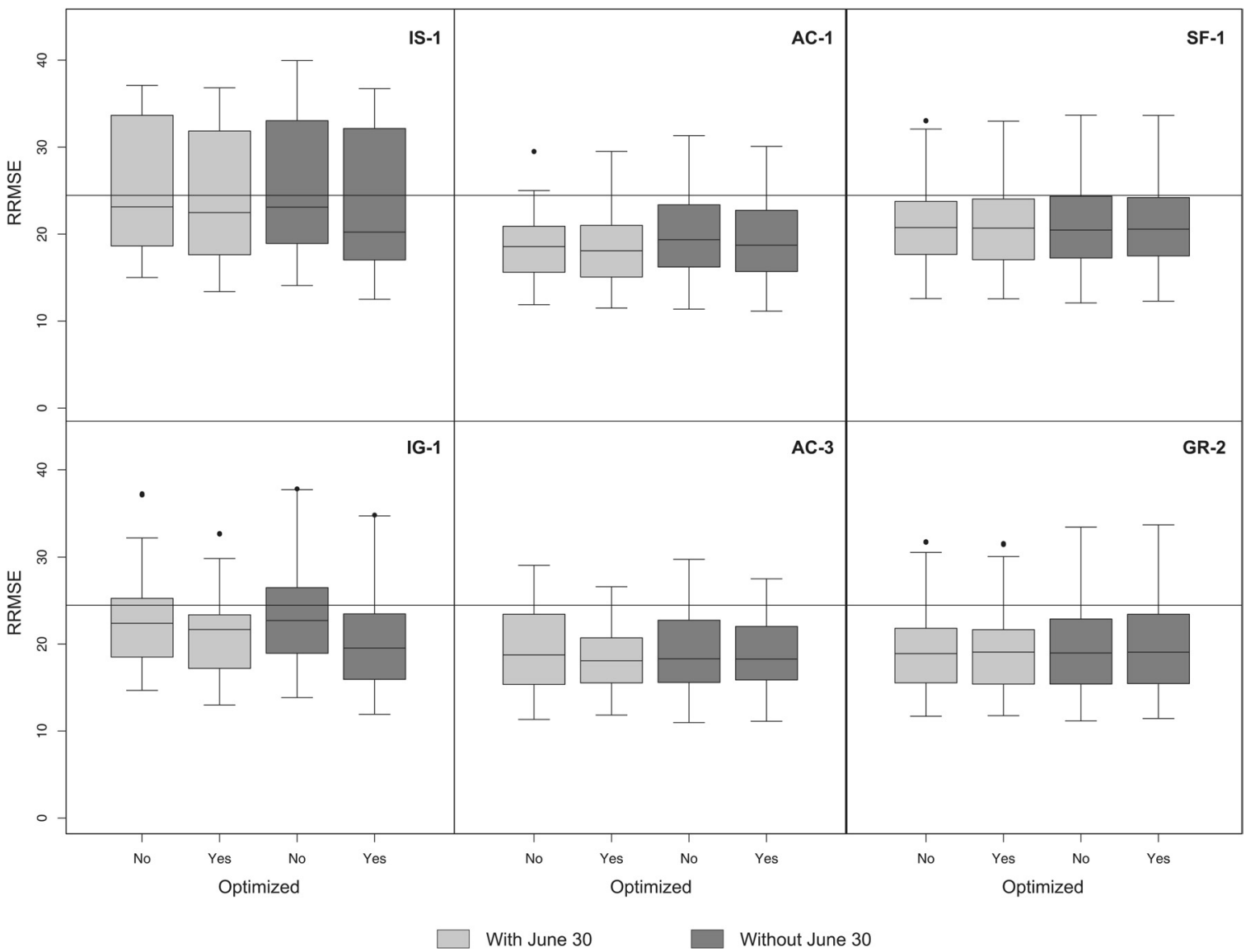

Fig. 7. Observed RRMSE of 6 estimators of the secondary production. The 4 boxplots of each panel correspond to optimized and non-optimized sampling schedules including June 30 or not. Each boxplot depicts variation over the remaining 18 (or 54) combinations of factors A, D, E, and F. The central line represents the median of all observed values of the RRMSE.

cohort contributes significantly to the total biomass or is otherwise deemed to be of primary importance (see, e.g., Bachelet and Yacine-Kassab, 1987), but such is not the case here.

3. Although estimation schemes designed to avoid negative productions may be justifiable in certain circumstances (see, e.g., Buchanan and Warwick, 1974; Hughes, 1978; Wildish and Peer, 1981), this practice is likely to increase bias and reduce precision when applied to mussel-like populations, as witnessed by the comparatively poor performance of IS-3, IS-4, IG-2, and AC-2. In particular, the proximity of sampling dates, combined with small sample sizes (e.g., 13 dates per year, 5 quadrats per sampling occasion), increases the frequency of negative productions between 2 successive dates; the replacement of many negative terms by zeros is a likely cause for the high positive bias observed for IS-4 (see Figs. 2 and 3).

4. Size-based methods are often considered much inferior to cohort-based estimators; see, e.g., Morin et al. (1987) or Plante and Downing (1990). Their unexpectedly good performance in the present context is likely attributable to the fact that the cohort production interval (CPI) was taken to be known. The CPI index generally refers to the length of time a species needs to complete its development; it was taken as equal to 8 in the current study. As already emphasized, e.g., by Waters (1979) and Benke (1984), an inaccurate knowledge of the life cycle of a species may lead to a much larger variability and bias in the estimation of its secondary production.

5. Although the bias and precision of all size-based methods were obviously affected by the (arbitrary) number $K$ of size classes, no trend could be ascertained. The difficulties associated with the selection of $K$ are akin to the choice of an appropriate number of bins in a histogram: whereas too few intervals is likely to mask genuine features of the data, too many will result in artificially empty bins and variation patterns that may be the effect of chance only. This phenomenon has long been recognized in secondary production estimation; see, e.g., Hamilton (1969), Lapchin and Neveu (1980). Many authors have observed that production values tend to decrease as the number of size classes gets larger, but there is only mitigated support for this conjecture in Table 3. 
Table 2. Observed PRB and RRMSE per quadrat of 6 methods of estimating secondary production.

\begin{tabular}{|c|c|c|c|c|}
\hline \multirow[b]{2}{*}{ Method } & \multicolumn{2}{|c|}{ PRB } & \multicolumn{2}{|c|}{ RRMSE } \\
\hline & $0.5 \mathrm{~mm}$ & $1 \mathrm{~mm}$ & $0.5 \mathrm{~mm}$ & $1 \mathrm{~mm}$ \\
\hline IS-1 & -3.66 & -3.84 & 26.03 & 26.02 \\
\hline IG-1 & 1.82 & 1.53 & 23.71 & 23.67 \\
\hline$A C-1$ & -7.80 & -7.97 & 20.04 & 20.08 \\
\hline AC-3 & 2.43 & -5.38 & 20.10 & 18.89 \\
\hline SF-1 & -10.37 & -8.01 & 22.08 & 22.20 \\
\hline GR-2 & -2.59 & -2.35 & 20.64 & 20.59 \\
\hline
\end{tabular}

The figures for the first 4 (cohort-based) methods are averaged over 36 combinations of factors A-D; the figures for the last 2 (size-based) methods are averaged over 108 combinations, due to the inclusion of factor $F$.

Observations and recommendations concerning sampling design-Of all factors tested, sampling schedule and effort (factors $\mathrm{A}$ and $\mathrm{D}$ ) were the most determinant in both bias and precision of production estimates. This is not surprising, since both factors increase sample size, as has already been observed in previous simulation studies; see, among others, Cushman et al. (1978), Lapchin and Neveu (1980), or Morin et al. (1987). For cohort-based methods, for example, the data are points on the Allen curve. Thus, while the addition of sampling dates gives a better idea of the shape of the curve, a larger number of quadrats on each sampling occasion improves the precision of point estimates on that curve; concerning the latter point, see Möller and Rosenberg (1982), Sköld et al. (1994), or Nithart (1998).

If sampling were limited to only a few quadrats per date (say, 5) and a few sampling occasions (say, 5), mass-specific growth-rate method GR-2 would appear to be the best option (cf. Figs. 4 and 5). It would also be an excellent choice if the experimenter could afford 15 quadrats per date and 13 dates per year. In the latter case, of course, the 6 methods considered in greater detail (IS-1, IG-1, AC-1, AC-3, SF-1, GR-2) perform quite well, so which one should be used is much less critical.

Surprisingly, the intensification of sampling dates during the growth season did not have a major effect, despite numerous suggestions to the contrary in the literature; see, e.g., Winberg et al. (1971), Chapman (1978), or Lapchin and Neveu (1980). For populations similar to that considered in this article, therefore, the sampling schedule could thus be adjusted as most convenient logistically, within reason. For example, no great loss in precision would ensue from concentrating sampling in the summer months for a species experiencing slow growth in the winter, so long as some sampling occurs in the latter season as well, to account for mortality. Although this is not shown herein, the MR methods that are based on mortality were found in the simulation to be less precise when using an optimized sampling design in the growth season only; see Cusson (2004) for details. With respect to the inclusion or exclusion of June 30 as the last sampling date, the simulation showed little effect to speak of, especially among the most recommendable methods.
Table 3. Observed PRB and RRMSE per quadrat of the 10 sizebased methods of estimating secondary production.

\begin{tabular}{lrrrrrrr}
\hline & \multicolumn{3}{c}{ PRB } & & \multicolumn{3}{c}{ RRMSE } \\
\cline { 2 - 4 } Method & \multicolumn{1}{c}{$\mathbf{1 0}$} & $\mathbf{1 5}$ & $\mathbf{3 0}$ & & $\mathbf{1 0}$ & $\mathbf{1 5}$ & $\mathbf{3 0}$ \\
\hline SF-1 & -5.38 & -10.12 & -12.06 & & 22.11 & 21.68 & 22.62 \\
SF-2 & -16.67 & -16.32 & -16.08 & & 25.99 & 24.87 & 25.38 \\
SF-3 & -6.59 & -10.75 & -12.26 & & 22.37 & 21.88 & 22.69 \\
GR-1 & -22.91 & -18.88 & -14.18 & & 28.17 & 25.40 & 22.88 \\
GR-2 & 1.21 & -2.14 & -6.47 & & 21.13 & 20.34 & 20.36 \\
GR-3 & 1.52 & 8.88 & 14.48 & & 21.67 & 24.59 & 28.20 \\
GR-4 & -22.13 & -19.09 & -15.88 & & 27.44 & 25.42 & 23.60 \\
MR-1 & 16.57 & 11.49 & 21.60 & & 39.73 & 31.32 & 37.80 \\
MR-2 & -31.85 & -29.66 & -29.64 & & 35.74 & 34.04 & 34.02 \\
MR-3 & -25.00 & -25.87 & -33.20 & & 32.05 & 32.94 & 38.23 \\
\hline
\end{tabular}

The figures are averaged over 72 combinations of factors A-E.

Although sieve screen aperture is an integral part of the experimental design and needs to be fixed a priori, this factor had relatively little effect in the present context; the only possible exception was method AC-3, where the less time-consuming use of a larger sieve tended to decrease the RRMSE, at the cost of a somewhat enlarged absolute bias. The general lack of effect of sieve screen aperture is in contrast with previous studies; see, e.g., Bachelet (1990), Bachelet and Yacine-Kassab (1987), Sola (1996), Dauvin (1988), and Sarda and Martin (1993). In some of this work, correction factors were used to account for coarse sieves because the use of a large mesh size $(1.0 \mathrm{~mm}$ instead of $0.5 \mathrm{~mm}$ ) was reported to induce a strong underestimation on small individuals and density of recruits in the population.

For populations similar to that which is considered in this simulation study, the lack of sieve effect is likely due to the fact that young individuals in the hypothetical mussel population account for a small proportion of the total production. When a small effect was observed, as in AC-3, it may be explained by a "leverage" effect. For these methods, a few missing individuals end up affecting the entire estimation, because in each case, a curve is fitted on the merged cohorts. For other methods, a similar effect may have been present within the first cohort, but it is attenuated by the small body size of the corresponding individuals.

\section{References}

Bachelet, G. 1990. The choice of a sieving mesh size in the quantitative assessment of marine macrobenthos: a necessary compromise between aims and constraints. Mar. Env. Res. 30:21-35.

Bachelet, G., and M. Yacine-Kassab. 1987. Integration of the postrecruitment phase in the population dynamics of the intertidal gastropod Hydrobia ulvae (Pennant). J. Exp. Mar. Biol. Ecol. 111:37-60.

Bayne, B. L., and C. M. Worrall. 1980. Growth and production of mussels Mytilus edulis from two populations. Mar. Ecol. Prog. Ser. 3:317-328. 
Benke, A. C. 1984. Secondary production of aquatic insects. In: The Ecology of Aquatic Insects. V. H. Resh and D. M. Rosenberg, Eds. Praeger, New York, pp. 289-322.

Brey, T. 2004. Empirical relations in aquatic populations. Version 4.04. In: Population Dynamics in Benthic Invertebrates: A Virtual Handbook. www.awi-bremerhaven.de/Benthic/ Ecosystem/FoodWeb/Handbook/main.html, accessed Jan. 31, 2006. Alfred-Wegener Institute, Bremerhaven, Germany.

Buchanan, J. B., and R. M. Warwick. 1974. An estimate of benthic macrofaunal production in the offshore mud of the Northumberland coast. J. Mar. Biol. Ass. U.K. 54:197-222.

Chapman, D. W. 1978. Production. In: Methods for Assessment of Fish Production in Fresh Waters. T. Bagenal, Ed. Blackwell, Oxford, pp. 202-217.

Crisp, D. J. 1984. Energy flow measurements. In: Methods for the Study of Marine Benthos. N. A. Holme and A. D. McIntyre, Eds. Blackwell, Oxford, pp. 284-372.

Cushman, R. M., H. H. Shugart Jr., S. G. Hildebrand, and J. W. Elwood. 1978. The effect of growth curve and sampling regime on instantaneous-growth, removal-summation, and Hynes/Hamilton estimates of aquatic insect production: a computer simulation. Limnol. Oceanogr. 23:184-189.

Cusson, M. 2004. Étude critique des méthodes d'estimation de la production secondaire macrobenthique marine. Doctoral dissertation, Département de biologie, Université Laval, Québec, Canada.

Cusson, M., and E. Bourget. 2005. Small-scale variations in mussel (Mytilus spp.) dynamics and local production. J. Sea Res. 53:255-268.

Dauvin, J.-C. 1988. Biology and population dynamics and production of Crustacea Amphipoda from the western English Channel: 3. Ampelisca typica (Bate). J. Exp. Mar. Biol. Ecol. 121:1-22.

Fréchette, M., and E. Bourget. 1985. Food-limited growth of Mytilus edulis L. in relation to the benthic boundary layer. Can. J. Fish. Aquat. Sci. 42:1166-1170.

Hamilton, A. L. 1969. On estimating annual production. Limnol. Oceanogr. 14:771-782.

Hughes, R. G. 1978. Production and survivorship of epizoites of the Hydroid Nemertesia antennina (L.). J. Mar. Biol. Ass. U. K. 58:333-345.

Krueger, C. C., and F. B. Martin. 1980. Computation of confidence intervals for the size-frequency (Hynes) method of estimating secondary production. Limnol. Oceanogr. 25:773-777.

Lapchin, L., and A. Neveu. 1980. The production of benthic invertebrates: comparison of different methods. Acta Oecologica / Oecologica Generalis 1:307-322.

Möller, P., and R. Rosenberg. 1982. Production and abundance of the amphipod Corophium volutator on the West coast of Sweden. Neth. J. Sea Res. 16:127-140.

Morin, A., T. A. Mousseau, and D. A. Roff. 1987. Accuracy and precision of secondary production estimates. Limnol. Oceanogr. 32:1342-1352.

Nithart, M. 1998. Population dynamics and secondary production of Nereis diversicolor in a North Norfolk Saltmarsh (UK). J. Mar. Biol. Ass. U. K. 78:131-143.

Plante, C., and J. A. Downing. 1990. Empirical evidence for differences among methods for calculating secondary production. J. N. Am. Benthol. Soc. 9:9-16.

Rigler, F. H., and J. A. Downing. 1984. The calculation of secondary production. In: A Manual on Methods for the Assessment of Secondary Productivity in Fresh Waters. J. A. Downing and F. H. Rigler, Eds. Blackwell, Oxford, pp. 19-86.

Sarda, R., and D. Martin. 1993. Populations of Streblospio (Polychaeta: Spionidae) in temperature zones: demography and production. J. Mar. Biol. Ass. U. K. 73:769-784.

Siegismund, H. R. 1982. Life cycle and production of Hydrobia ventrosa and Hydrobia neglecta (Mollusca: Prosobranchia). Mar. Ecol. Prog. Ser. 7:75-82.

Sköld, M., L. O. Loo, and R. Rosenberg. 1994. Production, dynamics and demography of an Amphiura filiformis population. Mar. Ecol. Prog. Ser. 103:81-90.

Sola, J. C. 1996. Population dynamics, reproduction, growth, and secondary production of the mud-snail Hydrobia ulvae (Pennant). J. Exp. Mar. Biol. Ecol. 205:49-62.

Waters, T. F. 1979. Influence of benthos life history upon the estimation of secondary production. J. Fish. Res. Bd. Can. 36:1425-1430.

Wildish, D. J., and D. Peer. 1981. Methods for estimating secondary production in marine Amphipoda. Can. J. Fish. Aquat. Sci. 38:1019-1026.

Winberg, G. G., K. Patalas, J. C. Wright, A. Hillbricht-Ilkowska, W. E. Cooper, and K. H. Mann. 1971. Methods for calculating productivity. In: A Manual on Methods for the Assessment of Secondary Productivity in Fresh Waters. W.T. Edmonston and G. G. Winberg, Eds. Blackwell, Oxford, pp. 296-317.

Submitted 31 May 2005

Revised 26 September 2005

Accepted 29 November 2005 